\title{
Circulating Current Suppression Strategy of Modular Multilevel Converter Based on Model Predictive Control
}

\author{
Liu kang ${ }^{1, *}$, Gao Guige ${ }^{2}$ \\ ${ }^{1}$ Shanghai DianJi University, Shanghai, 201306, China \\ ${ }^{2}$ Shanghai DianJi University, Shanghai, 201306, China
}

\begin{abstract}
Modular Multilevel Converter (MMC) has the characteristics of high voltage level and low switching frequency. The traditional modular multilevel converter circulating current control strategy mostly adopts the PI control principle, and the parameter setting is complicated and the accuracy is not high, and the control process is more difficult. Model predictive control strategy is the optimal control method based on the model in the existing time domain. This paper proposes a Model Predictive Control (MPC) method based on carrier phase-shifted pulse width modulation (PSC-PWM) to suppress the circulating current, and output the optimal modulation wave through model prediction. Compared with the traditional control strategy, this strategy is simple to implement, does not require complex tuning calculations, and combines with the traditional capacitor voltage equalization strategy to obtain the output modulation wave. A 7-level MMC simulation control system is built in MATLAB / SIMLINK to verify the theory, comparing with existing control methods, it can be concluded that the proposed method has high calculation efficiency, good control accuracy and strong robustness.
\end{abstract}

\section{Introduction}

Modular multilevel converter (MMC), as a new type of voltage source converter topology ${ }^{[1]}$, can be modularized and has good scalability. By increasing or reducing the number of series sub modules, the voltage level of application can be flexibly changed. However, due to its own topological structure characteristics, there will be problems such as unbalanced capacitor voltage and inter phase circulating current. In MMC control, the current inner loop control usually adopts AC current feedback control method. Since the AC side current can not represent the information of the internal circulating current in $\mathrm{MMC}$, it is necessary to design an additional circulating current controller. When the AC system is unbalanced, the $\mathrm{AC}$ current contains positive and negative sequence components, and the circulating current contains positive, negative and zero sequence components. The complexity of the control system will be greatly increased ${ }^{[2]}$, The phase to phase circulating current in MMC will directly lead to the current distortion of the bridge arm, aggravate the voltage fluctuation of the sub module and increase the system loss. The existing literatures mostly use the proportional or proportional resonant controller to suppress the circulating current. However, it is difficult to set the parameters of this method, and all the controllers are integrated to control, so the control method is complex. In [3], linear decoupling method is used to improve the control accuracy in two-phase rotating coordinate system, but the robustness of the system is not high. The sliding film control is added to improve the robustness, but the control is relatively complex. In [3], the quasi proportional resonance (QPR) controller is improved to suppress the circulating current based on the low pass filter (LPF) circulating current suppression strategy. In [4], based on the compound control of double coordinate system, the coordinate transformation of the second harmonic component is carried out to suppress the circulation. However, the method is too complex and has certain limitations.

In this paper, the model prediction method is used to suppress the circulating current. Because the bridge arm current contains $\mathrm{AC}$ and $\mathrm{DC}$ information, the bridge arm current is directly controlled to achieve the circulating current suppression effect. The rated reference value of the bridge arm current without the frequency doubling component is calculated. The actual current can track the reference current through the MPC prediction and tracking ability, so as to achieve the purpose of suppressing the circulating current. The principle is simple and the operator is simple Therefore, the circulation suppression can be achieved without complicated setting calculation.

\section{Mathematical model of MMC topology and traditional circulation suppression}

The topology of MMC is shown in Figure 1. Each phase is composed of two upper and lower bridge arms. Each arm is composed of $\mathrm{N}$ sub modules, equivalent reactance 
and equivalent resistance. $U_{(a, b, c)}$ is three-phase AC power supply, $L_{o}$ is the equivalent inductance and $R_{o}$ is the equivalent reactance. $u_{p j}$ is the voltage of upper and $u_{n j}$ is the lower bridge arms without equivalent resistance and inductance, $i_{p j}$ is the current of upper and $i_{n j}$ is the current lower bridge arms. Point $O$ is the zero reference point

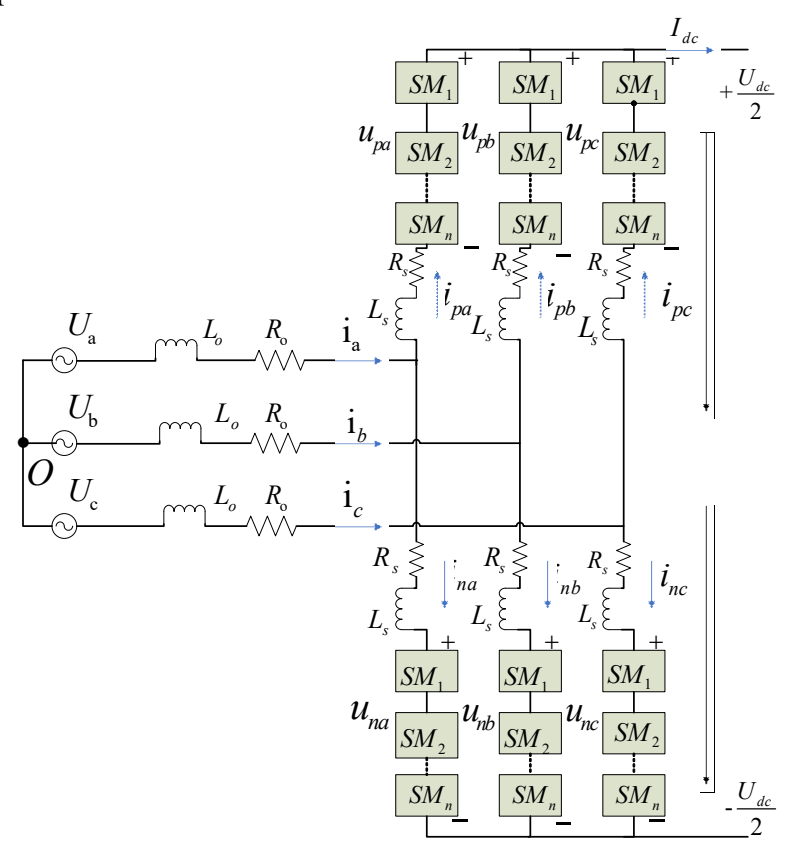

Fig. 1. topology of three phase MMC rectifier

According to the symmetrical structure of three-phase MMC, combined with figure 1 and KVL equation, phase $\mathrm{A}$ is analyzed as an example, and the following formula can be listed:

$$
\left\{\begin{array}{l}
U_{a}-L_{o} \frac{d i_{a}}{d t}-R_{o} \bullet i_{a}=\frac{U_{d c}}{2}+L_{s} \frac{d i_{p a}}{d t}+R_{s} i_{a}-u_{p a} \\
U_{a}-L_{o} \frac{d i_{a}}{d t}-R_{o} \bullet i_{a}=-\frac{U_{d c}}{2}+L_{s} \frac{d i_{n a}}{d t}+R_{s} i_{a}-u_{n a}
\end{array}\right.
$$

$$
\text { Order } U_{a}-L_{o} \frac{d i_{a}}{d t}-R_{a}=u_{o a}, u_{o a} \text { is the voltage of }
$$

rectifying point, According to formula (1), it is concluded that:

$$
\left\{\begin{array}{l}
L_{s} \frac{d i_{p a}}{d t}=u_{p a}+u_{o a}-R_{s} i_{p a}-\frac{U_{d c}}{2} \\
L_{s} \frac{d i_{n a}}{d t}=-u_{n a}+u_{o a}-R_{s} i_{n a}+\frac{U_{d c}}{2}
\end{array}\right.
$$

Next, take the current of the upper and lower bridge arms of phase a as an example to analyze the current

$$
\left\{\begin{array}{l}
i_{p a}=\frac{i_{a}}{2}+\frac{I_{d c}}{3}+i_{c i r} \\
i_{n a}=\frac{i_{a}}{2}-\frac{I_{d c}}{3}-i_{c i r}
\end{array}\right.
$$

Wher: $i_{c i r}$ is the AC frequency doubling component, which is the circulating current component to be suppressed. From (3), it can be seen that the bridge arm current contains $\mathrm{AC}$ side current and loop flow information. Therefore, if the bridge arm current is directly controlled and the reference value of bridge arm current without second harmonic component is tracked, the double frequency circulating current can be suppressed. The above is the basic voltage and current formula. The analysis method of other two phases is similar.

According to the principle of energy balance, the circulating current suppression method is to keep the voltage balance of the sub module capacitor, avoid the voltage difference between the bridge arms, and then play the role of circulating current suppression. However, the traditional circulation suppression needs a lot of PI control, so the parameter setting is more troublesome. The principle of the control method is simple, but the actual calculation is large. As shown in Figure 2, the traditional circulation suppression strategy is shown.

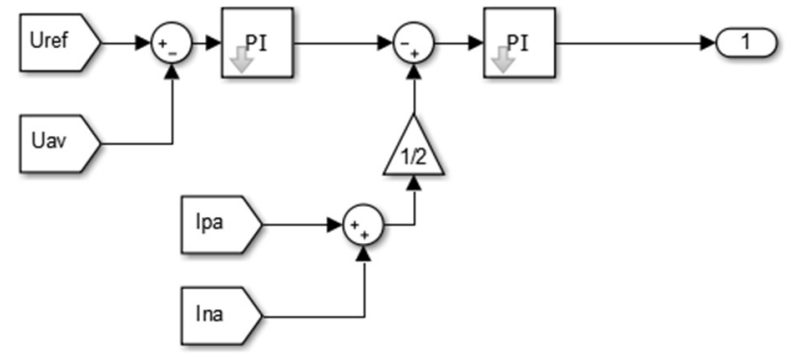

Fig. 2. traditional circulation suppression chart

In the figure, $U_{r e f}$ and $U_{a v}$ are the reference value and average value of capacitor voltage, $i_{p a}$ and $i_{n a}$ are the current of upper and lower bridge arms. The outer loop voltage in the diagram adopts PI regulator, the average capacitor voltage $U_{a v}$ in the control phase unit tracks the reference value $U_{r e f}$ of the capacitor voltage, and the output of the voltage outer loop is used as the current reference value; the current inner loop also adopts PI The regulator is used to control the circulating current to track the change of the reference value of the given circulating current, and its output is used as the regulating value of capacitor voltage balance control.

\section{Circulation suppression based on MPC}

MPC is different from the above-mentioned frequency domain control, which requires complex controller calculation. MPC control based on time domain can track complex composite signals. Based on the characteristics of MMC circulation, this paper designs a circulating current suppression strategy based on MPC. Taking phase a as an example, the sampling period of the system is the discrete model of MMC rectifier is obtained by discretizin (3). 


$$
\left\{\begin{array}{l}
i_{p a}(k+1)=\left(1-\frac{R_{s} \cdot T_{s}}{L_{s}}\right) i_{p a}(k)+\frac{T_{s}}{L_{s}}\left(u_{p a}(k)+u_{o a}(k)-\frac{U_{d c}(k)}{2}\right) \\
i_{n a}(k+1)=\left(1-\frac{R_{s} \cdot T_{s}}{L_{s}}\right) i_{n a}(k)+\frac{T_{s}}{L_{s}}\left(-u_{n a}(k)+u_{o a}(k)+\frac{U_{d c}(k)}{2}\right)
\end{array}\right.
$$

Where: $i_{p a}(k)$ and $i_{n a}(k)$ are the current values at $\mathrm{k}$ time of upper and lower bridge arms of phase $\mathrm{A}, u_{o a}(k)$ and $U_{d c}(k)$ are the voltage of parallel point and DC bus at $\mathrm{k}$ time, and $\mathrm{a}$ and $\mathrm{B}$ are the predicted current values at $\mathrm{k}$ time.

From (4), the prediction model of the upper and lower arms of phase a can be obtained as follows:

$$
\left\{\begin{array}{l}
i_{p a}(k+1 / k)=\left(1-\frac{R_{s} \cdot T_{s}}{L_{s}}\right) i_{p u}(k)+\frac{T_{s}}{L_{s}}\left(u_{p u}(k / k)+u_{o c}(k)-\frac{U_{d k}(k)}{2}\right) \\
i_{n a}(k+1 / k)=\left(1-\frac{R_{s} \cdot T_{s}}{L_{s}}\right) i_{n a}(k)+\frac{T_{s}}{L_{s}}\left(-u_{n k}(k / k)+u_{\alpha a}(k)+\frac{U_{d k}(k)}{2}\right)
\end{array}\right.
$$

Where: $\mathrm{K}+1 / \mathrm{K}$ in brackets represents the system state predicted at $\mathrm{K}+1$ time at current $\mathrm{K}$ time, $u_{p a}(k)$ and $u_{n a}(k)$ are the optimal output modulation voltage command value of upper and lower bridge arms after circulating current suppression.

In order to improve the accuracy of prediction, feedback correction is designed as:

$$
\left\{\begin{array}{l}
h_{p j}=\lambda_{1}\left(i_{p j}(k)-i_{p c . j}(k / k-1)\right. \\
h_{n j}=\lambda_{2}\left(i_{n j}(k)-i_{n c . j}(k / k-1)\right.
\end{array}\right.
$$

Where $\mathrm{j}=\mathrm{a}, \mathrm{b}, \mathrm{c}$ are three-phase, $\lambda_{1}$ and $\lambda_{2}$ are feedback coefficients; $i_{p c, j}(k / k-1)$ and $i_{n a}(k / k-1)$ are prediction values of bridge arm current at k-1 time based on feedback term at upper and lower arms respectively.

The current prediction model of three-phase upper and lower bridge arms based on feedback phase is as follows:

$$
\left\{\begin{array}{l}
i_{p j}(k+1 / k)=x i_{p j}(k)+y\left(u_{p j}(k / k)+u_{o j}(k)-\frac{U_{d c}(k)}{2}\right)+h_{p j} \\
i_{n j}(k+1 / k)=x i_{n j}(k)+y\left(-u_{n j}(k / k)+u_{o j}(k)+\frac{U_{d c}(k)}{2}\right)+h_{n j}
\end{array}\right.
$$

In (7): $x=\left(1-\frac{R_{s} \cdot T_{s}}{L_{s}}\right), y=\frac{T_{s}}{L_{s}}$,In the actual prediction, since the current reference value at $\mathrm{t}+\mathrm{TS}$ time is calculated based on the voltage sampling value at time $t$, while the reference value of phase current contains the AC quantity of phase information, there will be a lag error between the actual current value and the reference value determined by the sampling interval TS. If the phase current $i_{j}^{*}=I \sin \left(\omega t+\varphi_{j}\right)$ is known from reference [3], the error is:

$$
\begin{aligned}
& \Delta i_{j}=\left[1-\cos \left(\omega T_{s}\right)\right] \cdot I \sin \left(\omega t+\varphi_{j}\right) \\
& +\sin \left(\omega T_{s}\right) I \cos \left(\omega t+\varphi_{j}\right) \\
& \approx I \cos \left(\omega t+\varphi_{j}\right) \sin \left(\omega T_{s}\right)
\end{aligned}
$$

Where: $j=a, b, c$ and $\omega$ are grid voltage angular frequency. The difference between $\varphi_{a}, \varphi_{b}$ and $\varphi_{c}$ is 1200 . According to the ideal transmission process with power, we can get the following results:

$$
I_{d c}^{*}=\frac{P}{U_{d c}}
$$

In order to suppress the frequency doubling component of the circulating current, the reference value of the bridge arm current can be obtained by combining the above formula:

$$
\left\{\begin{array}{l}
i_{p j}^{*}=\frac{i_{j}}{2}+\frac{i_{d c}^{*}}{3}+\frac{\Delta i_{j}}{2} \\
i_{n j}^{*}=\frac{i_{j}}{2}-\frac{i_{d c}^{*}}{3}+\frac{\Delta i_{j}}{2}
\end{array}\right.
$$

The objective function is designed to solve the optimal control quantity of the upper and lower bridge arms at $\mathrm{k}$ time. The objective function should satisfy two points: first, it can accurately track the current command value, that is, the predicted value of current at $\mathrm{K}+1$ time is as close as possible to the actual value; secondly, the stability of the control system is guaranteed, that is, the control braking action is not too large, so the objective function needs to add a constraint on the control quantity. Therefore, the objective function can be designed:

$$
\left\{\begin{array}{l}
J_{1}(u)=\varepsilon_{1}\left(i_{p j}^{*}(k+1)-i_{p j}(k+1)\right)^{2}+\omega_{1}\left(u_{p j}(k)\right)^{2} \\
J_{2}(u)=\varepsilon_{2}\left(i_{n j}^{*}(k+1)-i_{n j}(k+1)\right)^{2}+\omega_{2}\left(u_{n j}(k)\right)^{2}
\end{array}\right.
$$

Where $\varepsilon_{1}$ and $\varepsilon_{2}$ are the current coefficients of the upper and lower bridge arms, $\omega_{1}$ and $\omega_{2}$ are the restraint coefficient of the upper and lower bridge arms. To solve (11), the first term of the optimal solution sequence is the optimal output modulation voltage of the upper and lower bridge arms.

\section{Simulation verification}

As shown in Figure 3, the phase-shift carrier control strategy based on model prediction is mainly divided into three parts: Calculation of current reference value, model prediction module and pulse signal generation module. In the current reference value calculation part, in order to suppress the frequency doubling component of the circulating current, the value is set to zero. It is worth mentioning that only the model predictive control is used to control the energy imbalance between the bridge arms, so the upper system energy balance control is needed. 


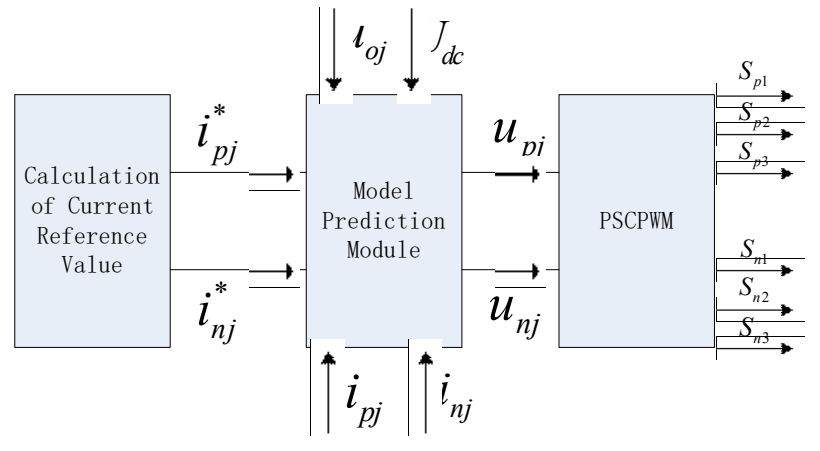

Fig. 3. model predictive control

Table 1 shows the parameter settings of the modular multilevel converter. With the same parameter settings, the circulating current suppression effect of traditional control strategy and model predictive control is compared. In this paper, a 7-level modular multilevel rectifier is built in SIMLINK to verify the correctness of the control strategy.

Table 1 Model simulation parameters.

\begin{tabular}{|c|c|}
\hline Parameter & Given Value \\
\hline Carrier frequency & $2000 \mathrm{~Hz}$ \\
\hline Capacitance & $10 \mathrm{mF}$ \\
\hline Bridge arm resistance & $0.05 \Omega$ \\
\hline Bridge arm inductance & $4 \mathrm{mH}$ \\
\hline AC side inductance & $3 \mathrm{mH}$ \\
\hline AC side resistance & $0.25 \Omega$ \\
\hline
\end{tabular}

As shown in Fig. 4, the actual current of the bridge arm follows the command value of the reference current to achieve the desired waveform diagram, which can prove the correctness of the experimental parameters and the feasibility of the proposed method.

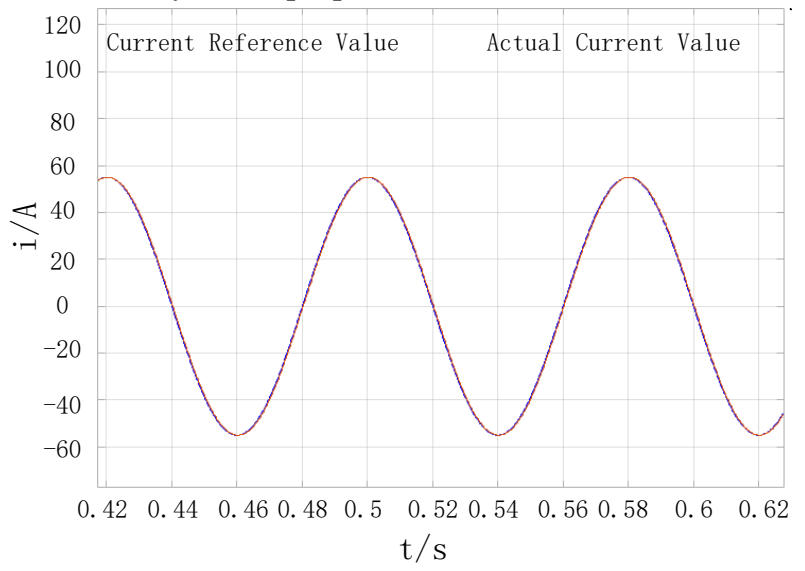

Fig. 4. Phase a upper bridge arm current

Fig 5 shows the FFT diagram of the traditional control strategy. From the figure, we can see that the total harmonic distortion (THD) is $24.02 \%$, Fig 6shows the FFT analysis chart of the predicted value of the model. THD value of the model prediction model is $0.1 \%$, and the output waveform quality is very high. It can be seen that the model predictive control can play a good role in high-order harmonic suppression, and the suppression effect on the circulation is obviously better than that of the traditional circulation control strategy, and the correctness of the control strategy can be seen.

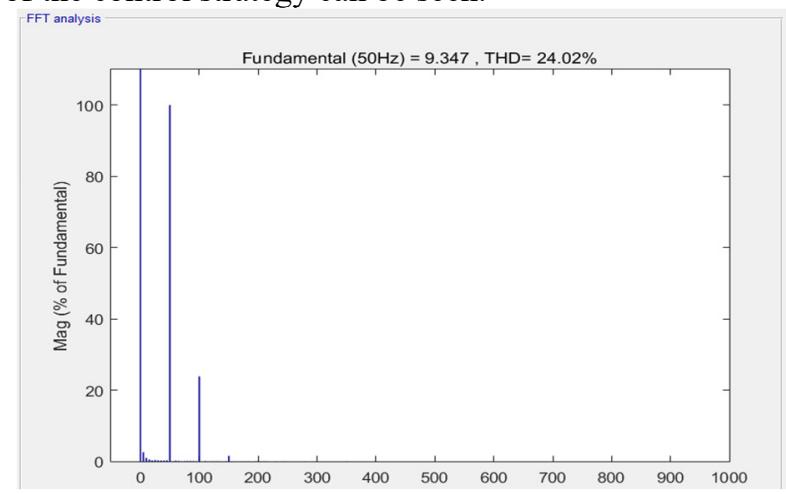

Fig. 5. FFT analysis of control strategy

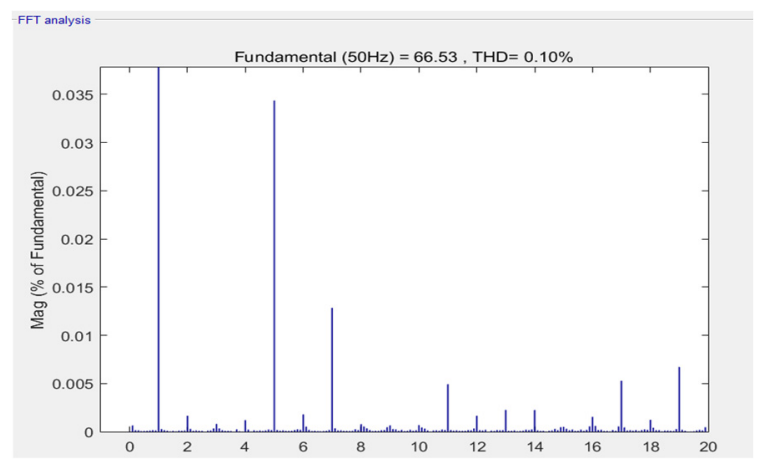

Fig. 6. FFT analysis of model predictive control

\section{Conclusion}

In this paper, a circulation suppression strategy for MMC based on model prediction is proposed. The correctness and effectiveness of the control strategy proposed in this paper are verified by simulation and data analysis. The model predictive control does not need complex setting calculation, and avoids the addition of a large number of PI and PR controllers. In this paper, we need to establish the corresponding target function, which can track the multi band composite signal with high control accuracy and good circulation suppression effect. The modular multilevel converter based on model predictive control has the advantages of simple control method, good output waveform quality and low harmonic content, which is more suitable for engineering application. However, the discrete formula of model predictive control strategy has some errors, and the amount of error can be optimized to further improve the control accuracy. And this paper only carried out theoretical analysis and verification, and did not put into the actual project to verify.

\section{Reference}

1. Pu L, Yue W, Wulong C. (2014)Study on optimal model predictive control strategy of modular multilevel converter[J]. Acta Sinica, 34:6380-6388.

2. Huancheng L,Zhixin W. (2016)A novel hybrid control strategy for modular multilevel converter 
based on model predictive control[J]. Chinese Journal of electrical engineering, 36:1877-1884.

3. Pengwu W, Xiang C, pengwu W. (2013)Three phase decoupling second harmonic current suppression algorithm for mmc-hvdc[J]. Power system automation, 37:47-52.

4. Wanyun L,Shoudao H, Jingwei H. (2015)Research on redundant operation control strategy of modular multilevel converter $[\mathrm{J}]$. Power electronics technology, 49:33-40.

5. Xiaofeng Y, Zejie L, qionglin Z. (2018)Circulating current suppression strategy of MMC based on virtual impedance sliding mode control [J]. Chinese Journal of electrical engineering, 38:127-138. 